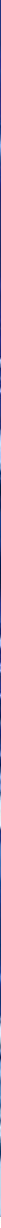

\title{
VACUUM SCIENCE AND TECHNOLOGY AT CERN
}

I José Miguel Jiménez and Paolo Chiggiato, CERN, Geneva, Switzerland - https://doi.org/10.1051/epn/2020404

Vacuum is essential in particle accelerators. Low gas density allows charged particles beams to circulate without excessive losses. Indeed, beam losses are detrimental for instrumentation; they increase induced radioactivity, background noise in particle detectors, and beam-induced heat loads to cryogenic equipment.

$\triangle$ View of

the central beam pipe of the LHCb, one of the four gigantic LHC's experiments.

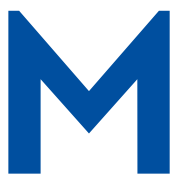
oreover, the interaction of beams with gas provokes beam instability and beam-size growth leading to a reduced probability of collisions in detectors. Finally, vacuum is necessary to avoid electrical breakdown in high-voltage devices and serves as an excellent commonly used thermal insulator in cryogenics. CERN has one of the world largest vacuum systems in operation [1]. Along $127 \mathrm{~km}$ of vacuum vessels, the pressure requirements cover a large range, from $10^{-6} \mathrm{mbar}$ in the first stage of linear accelerators down to $10^{-15} \mathrm{mbar}$ in the antimatter experiments. The Large Hadron Collider (LHC) is the best example of CERN's prowess in vacuum technology [2]. Building its vacuum system required more than 250,000 welded joints and 18,000 vacuum seals, thousands of pumps, valves, pressure gauges, PLC and controllers that need continuous monitoring with highest standards of reliability.

The beams as the main cause of gas release

Materials in vacuum release gas spontaneously. In particle accelerators, the beams stimulate additional desorption that can be the dominant gas source. Beam-stimulated desorption occurs directly due to beam losses or indirectly by emitting synchrotron light and accelerating electrons and ions created by residual-gas ionization. Bombardment of surfaces by such particles results in gas desorption. In the $\mathrm{LHC}, 7-\mathrm{TeV}$ proton beams emit synchrotron radiation with a critical energy around $40 \mathrm{eV}$, largely enough 
to extract photoelectrons and induce desorption. All these phenomena contribute to the degradation of the static vacuum, generating the so-called dynamic vacuum. For cost reasons, accelerator vacuum systems are never designed to cope with full beam performance on day one but rely on performance ramping-up scenarios. The impingement of photons, electrons and ions cleans the surface and provokes surface modifications that cause the reduction of desorption yields. As an example, a dose of $10^{-2} \mathrm{C} . \mathrm{mm}^{-2}$ of $300-\mathrm{eV}$ electrons reduces the desorption yield of $\mathrm{H}_{2}$ by roughly one orders of magnitude. Accelerators affected by stimulated desorption are initially run at progressively increasing beam current so that the dose of impinging particles increases without unduly rising beam losses. In the jargon, we call this process 'scrubbing run' leading to 'surface conditioning. Typical surface transformations are reduction of hydroxides and graphitization of hydrocarbon contamination [3]. Such transformations have also a beneficial effect on the secondary electron yield of the exposed surfaces and, consequently, mitigate electron multipacting phenomena!

\section{Distributed pumping}

Spontaneous and beam-induced gas release are distributed along the ring of the accelerators, while vacuum pumps are installed in precise positions and act locally. The difference in the distribution of gas source and pumping action generates parabolic pressure profiles with a maximum in between two consecutive pumps. The pressure bump is amplified when the beam-pipe conductance is small, i.e. for small diameters and long length. Such an issue has been removed with the development of distributed pumping. An innovative solution based on non-evaporable getter (NEG) film coatings was developed in the late nineties at CERN [4]. The vacuum chamber is coated with a $\mu$ m-thick Ti-Zr-V thin film. The film is activated in the accelerator by heating the chamber to temperatures of at least $180^{\circ}$ for $24 \mathrm{~h}$. The activation process dissolves the native oxide layer into the bulk of the film. When back at room temperature, the surface is very clean and pumps most of the residual gas species. Activated NEG coatings provide also lower stimulated desorption and secondary electron yields, therefore significantly reducing the conditioning time and electron multipacting. About 1400 vacuum chambers, around 6 $\mathrm{km}$ of beam pipes, were NEG coated for the LHC and are since 2008 operating with beams [5]. Those located in the centre of the four gigantic LHC's experiments, made of beryllium, are the most demanding in terms of vacuum performance and mechanical properties. The implementation of NEG thin-film coatings has allowed innovative design for the new generation of synchrotron light sources. Thanks to such materials, it is possible to pump inside long vacuum chambers having a few-mm diameter. Conductance limitation is no longer a showstopper! This solution is considered for new low-emittance synchrotron radiation sources. Among them, MAX IV (Lund, $\mathrm{SE})$ is the archetype [6].

\section{Beam screen, heat load and carbon thin film}

NEG coating can be applied only in bakeable sections of accelerators operating at room temperature. In the case of the LHC, about $40 \mathrm{~km}$ of beam pipes are inserted in superconducting magnets operating at $1.9 \mathrm{~K}$ with superfluid helium. During operation, gas pumping relies only on adsorption on the cold surfaces. The cryogenic temperature compromises the 'surface conditioning' as the desorbed gasses are immediately re-adsorbed on the nearby surfaces. The problem is circumvented separating the surface where photons and electrons strike from the one where the most critical gas, i.e. hydrogen, is condensed. This is obtained by inserting in the cold bore of the magnets, which is at $1.9 \mathrm{~K}$, an additional pipe, called beam screen (Fig. 2), kept at a slightly higher temperature between 10 and $20 \mathrm{~K}$. Gas molecules can reach the coldest surface through mm-wide pumping slots where they are screened from beam-induce effects. Among several other functions, the beam screen has also the role of intercepting at higher temperatures than $1.9 \mathrm{~K}$ the heat load transferred from the beam to the cryogenic system, therefore reducing the electric energy consumption of the cryogenic system.

Since 2014, the LHC cryogenic plants have experienced an unexpected high thermal load in the beam-screen circuits. The excessive heat is not uniformly distributed along the ring; only four octants are affected. Today, there is enough evidence that the issue is due to secondary electrons accelerated by the proton beams and multiplied by the beam-screen surfaces, the so-called electron cloud phenomenon. CERN has developed a solution that has a mitigation effect on electron multipacting [7]. Since

V FIG. 1:

The beam screen prototype for the Q2 quadrupole magnets of the final focusing system for High-Luminosity LHC.

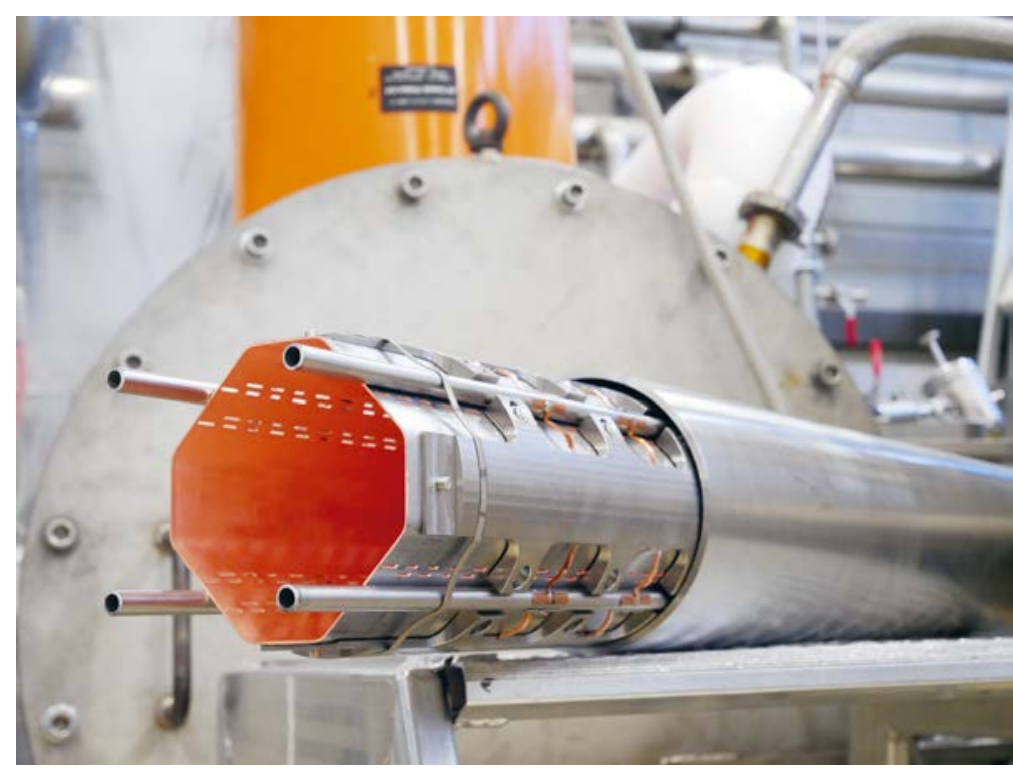


2008, sputtered graphitic-like amorphous carbon coatings have been studied and recently retrofitted in the accelerator beam pipes [8]. Fifty nanometres of this material are enough to decrease the maximum secondary electron emission yield below one and, consequently, cancel the electron multipacting. A similar reduction of the secondary electron emission can be achieved increasing the surface roughness by laser. Emitted secondary electrons are intercepted by the corrugated surface preventing them from being accelerated by the proton beams. This process was proposed by two British institutes [9] and it is under development for implementation in accelerators at CERN.

\section{Future challenges}

The present trend shows that surface modification and monitoring are key challenges for improving vacuum systems of high energy and intensity particle accelerators. In the future, four additional breakthroughs need to be addressed.

Cost containment. With the increasing size large scientific instruments such as the gravitational waves detectors or future colliders, there is strong demand for cost-optimised solutions that challenge present materials, surface treatments, pumps and operation procedures.

Miniaturisation, primarily for electron accelerators. The search for ultimate emittance (i.e. smaller transverse beam sizes) requires magnets with very small apertures, putting the beam-pipe walls as close as technically possible to the beams. Development of vacuum technology at the $\mathrm{mm}$-size diameter requires inventiveness for alternative manufacturing processes, pumping and pressure measurement.

To cope with high energy and intensity beams. Both high energy and high intensity may become a showstopper in accelerators like the High-Luminosity LHC, which will run in 2027, and the Future Circular Collider (FCC-hh), which is at the study level. The higher induced radioactivity, in particular nearby the experimental areas, would require that vacuum systems become compatible with robotic interventions. Preliminary works have been launched to design systems that can be installed, dismounted, and leak tested remotely. Innovative joints based on shape memory alloys [10] have been recently developed for a complete remote handling of junctions between vacuum chambers.

Finally, the quest for "absolute" vacuum. Experimental physics requires unprecedented low residual gas densities to address new types of experiments. Recently, the request for gas density of the order of one $\mathrm{H}_{2}$ molecule per $\mathrm{cm}^{3}$ has been formulated by the PUMA experiment, which aims to interact radioactive ions with stored antiprotons [11]. Gas density simulations, choice of materials, mechanical vibrations, transport constraints, and pressure measurement must be analysed in detail and all together to validate the feasibility of such experiments.
Undoubtedly, and through decades, the use of vacuum in large-scale scientific instruments has generated an impressive progress in Vacuum Science and Technology. The recently approved European Strategy for Particle Physics opens the path for new developments to respond to the needs of high energy and nuclear particle physics, and potential technology spin-off that may serve astrophysics and gravitational wave experiments.

\section{About the authors}

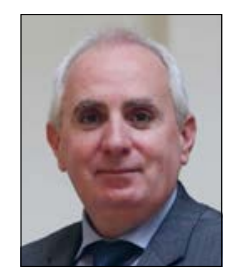

José Miguel Jiménez and Paolo Chiggiato are CERN's Technology department head and Vacuum Surfaces and Coating group leader, respectively. They have an extensive experience in vacuum technology with focus on accelerators' operation and surface mod-

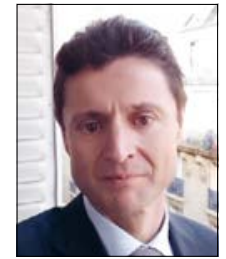
ifications. They gave an important contribution to the understanding of surface related phenomena in particle accelerators, e.g. beam-induced dynamic effects such as electron cloud and chemical pumping. Both contributed to the design, installation, commissioning and operation of the Large Hadron Collider (LHC) vacuum systems, and are actively involved in technology transfer programmes and international collaborations.

\section{References}

[1] J. M. Jiménez, Vacuum 84, 2 (2009)

[2] P. Chiggiato, "CERN's prowess in nothingness," CERN COURIER, 1 June 2018. https://cerncourier.com/a/cerns-prowess-innothingness/

[3] R. Cimino, M. Commisso, D. R. Grosso, T. Demma, V. Baglin, R. Flammini and R. Larciprete, Phys. Rev. Lett. 109, 064801 (2012).

[4] C. Benvenuti, P. Chiggiato, P. Costa Pinto, A. Escudeiro Santana, T. Hedley, A. Mongelluzzo, V. Ruzinov and I. Wevers, Vacuum $601-2,57$ (2001).

[5] P. Chiggiato and P. Costa Pinto, Thin Solid Films 515, 382 (2006).

[6] CERN Bulletin, 25 June 2016, "Vacuum chambers full of ideas for the Swedish synchrotron" https://home.cern/news/news/ engineering/vacuum-chambers-full-ideas-swedish-synchrotron

[7] CERN Bulletin, 29 February 2016, “A new type of coating to chase the clouds away", https://cds.cern.ch/journal/ CERNBulletin/2016/09/News\%20Articles/2133913?In=en

[8] CERN Bulletin, 1 October 2019, "LS2 Report: Dissipating the electron clouds", https://home.cern/news/news/accelerators/ Is2-report-dissipating-electron-clouds

[9] R. Valizadeh, O. B. Malyshev, S. Wang, S. A. Zolotocskaya, W. A. Gillespie and A. Abdolvand, Appl. Phys. Lett. 105, 231605 (2014)

[10] CERN Bulletin, 11 April 2016, "Shape memory" material provides a solution for the HL-LHC", http://cds.cern.ch/journal/ CERNBulletin/2016/16/News\%20Articles/2144535?ln=en

[11] A. Obertelli, "Experiment Proposal for PUMA: antiprotons and radioactive nuclei", 2019, http://cds.cern.ch/record/2691045/ files/SPSC-P-361.pdf 